\title{
Prevalência e fatores associados ao uso de álcool durante a gestação em uma maternidade de Goiás, Brasil Central
}

\author{
Prevalence and factors associated with alcohol use \\ during pregnancy in a maternity hospital in Goiás, Central Brazil
}

\author{
Vanessa Alves Guimarães ${ }^{1}$ \\ Kelly Silveira Fernandes ${ }^{1}$ \\ Roselma Lucchese ${ }^{1}$ \\ Ivânia Vera ${ }^{1}$ \\ Bruno César Teodoro Martins ${ }^{1}$ \\ Thiago Aquino de Amorim ${ }^{1}$ \\ Rafael Alves Guimarães ${ }^{1}$
}

${ }^{1}$ Universidade Federal de Goiás. Av. Dr. Lamartine Pinto de Avelar 1120, Setor Universitário. 75704020 Catalão GO Brasil. rafaelalvesg5@gmail.com

\begin{abstract}
The scope of this article is to estimate the prevalence and factors associated with alcohol use during pregnancy. It involved a cross-sectional study in a sample of 361 pregnant women in a reference service for gynecological and prenatal care. The data related to socio-demographic characteristics, alcohol use and potential associated factors were collected through face-to-face interviews. Poisson regression with robust variance was used to identify factors associated with the outcome analyzed. The consumption of alcohol in the sample was $17.7 \%$ (95\% CI: $95 \%$ CI: $14.1 \%$ to $22.0 \%)$. A history of pre-gestational or gestational diabetes, suicidal ideation and tobacco use in the last 30 days was associated with alcohol use during pregnancy $(p<0.05)$. The study showed a high prevalence of alcohol use during the current pregnancy and its association with important factors. Actions such as screening for alcohol and advice on problems associated with the use of this substance, especially during the prenatal period, can contribute to effective reduction of alcohol use in pregnant women and related maternal and fetal injuries.
\end{abstract}

Key words Alcohol consumption, Pregnant women, Risk factors
Resumo O objetivo deste artigo é estimar a prevalência e os fatores associados ao uso de álcool durante a gestação. Estudo de corte transversal em uma amostra de 361 gestantes de um serviço de referência à assistência ginecológica e pré-natal. Os dados relacionados às características sociodemográficas, uso de álcool e potenciais fatores associados foram coletados por meio de entrevista face a face. Análise de regressão de Poisson com variância robusta foi utilizada para verificar os fatores associados ao desfecho analisado. O consumo de álcool na amostra estudada foi de 17,7\% (IC 95\%: IC 95\%: 14,1-22,0\%). Antecedentes de diabetes pré-gestacional ou gestacional, ideação suicida e uso de tabaco nos últimos 30 dias foi associado ao uso de álcool durante a gestação $(p<0,05)$. O estudo apontou alta prevalência de uso de álcool na gestação atual e a sua associação com importantes fatores. Ações como rastreio para o álcool e aconselhamentos sobre os problemas associados ao uso dessa substância principalmente no pré-natal podem contribuir para redução efetiva ou anulação do seu uso em gestantes e agravos materno-fetais relacionados.

Palavras-chave Consumo de bebidas alcoólicas, Gestantes, Fatores de risco 


\section{Introdução}

O uso de álcool durante a gestação representa um grave problema de saúde pública. Esse comportamento tem sido associado a desfechos maternofetais, como a restrição do crescimento intrauterino, descolamento de placenta, abortos e anomalias congênitas (por exemplo: microcefalias e microftalmia $)^{1,2}$. A síndrome alcoólica fetal (SAF) corresponde a uma das principais consequências da exposição do feto ao álcool, sendo caracterizada por deficiência no crescimento, disfunções no desenvolvimento do sistema nervoso, entre outras alterações ${ }^{3}$. Além disso, crianças expostas ao álcool no período fetal apresentam maiores chances de desenvolvimento de transtornos mentais (transtorno comportamental relacionado ao uso de substâncias psicoativas e depressão) e comportamentais (personalidade antissocial e hiperatividade) na adolescência e vida adulta ${ }^{1,2,4,5}$.

Alguns estudos têm mostrado elevadas taxas de uso de álcool durante a gestação em várias localidades geográficas. Em países desenvolvidos, investigações têm mostrado prevalências de 6,0\% na Suécia ${ }^{6}, 10,2 \%$ nos Estados Unidos da América (EUA) $)^{7}, 10,8 \%$ no Canadá ${ }^{8}, 40,0 \%$ na Austrália, 56,0\% na Nova Zelândia e 75,0\% na Inglaterra ${ }^{9}$. Estimativas do uso de álcool em gestantes em países em desenvolvimento são escassas. No entanto, alguns estudos têm demonstrado altos índices na África do Sul $(34,9 \%)^{10}$, Uruguai $(63,1 \%)^{11}$ e Argentina $(75,2 \%)^{12}$. No Brasil, um estudo multicêntrico conduzido em 5.539 gestantes de ambulatórios de hospitais públicos de Manaus, Fortaleza, Salvador, Rio de Janeiro, São Paulo e Porto Alegre estimou uma prevalência de uso de álcool durante a gestação de $34,4 \%{ }^{13}$.

Uma rede complexa de variáveis sociodemográficas, comportamentais e familiares é associada ao uso de álcool na gestação. Os fatores sociodemográficos com maior intersecção com esse comportamento incluem idade mais elevada e status socioeconômico desfavorável (renda e escolaridade baixa) ${ }^{13,14}$. Determinantes comportamentais são representados, sobretudo, pelo uso simultâneo de tabaco e/ou drogas ilícitas ${ }^{15,16}$. Variáveis familiares incluem antecedentes familiares de transtornos mentais ${ }^{17}$ e/ou com história de uso de álcool e relações familiares disfuncionais ${ }^{4}$. Além disso, fatores como presença de transtornos mentais, ideação suicida ${ }^{18,19}$, gravidez não planejada ${ }^{20}$, infecções sexualmente transmissíveis (IST) prévias ${ }^{21}$ e exposição a situações de violência $^{1}$ têm sido associados ao consumo de álcool durante a gestação.
No Brasil, são poucos os estudos sobre a epidemiologia do uso de álcool durante a gestação, sendo a maioria, conduzidos na região sudeste do País ${ }^{22-25}$. Além disso, há uma lacuna na literatura sobre os preditores de uso de álcool em gestantes, especialmente no Brasil, dados que podem subsidiar políticas e ações de prevenção e controle dessa substância nessa população. Assim, o presente estudo objetivou estimar a prevalência e os fatores associados ao uso de álcool durante a gestação.

\section{Métodos}

Entre maio de 2014 e junho de 2015, um estudo de corte transversal foi conduzido em uma amostra não probabilística de gestantes atendidas em uma unidade de referência em assistência ginecológica e pré-natal de Goiás, Região Centro -Oeste do Brasil. Foram incluídas gestantes em qualquer idade gestacional e que se encontravam em acompanhamento de pré-natal na instituição do estudo. Mulheres em estado aparente de confusão mental ou com transtornos mentais que inviabilizasse sua participação foram excluídas.

Para o cálculo amostral, considerou-se o número de gestantes cadastradas na instituição durante o período de coletas de dados. Assim, o número necessário para compor a amostra foi de 293 mulheres, considerando uma população de 566 gestantes registradas, poder estatístico de $80 \%(\beta=20 \%)$, nível de significância de $95 \%$ ( $\alpha$ $=0,05)$, efeito de desenho de 3.0, 30\% de possíveis para possíveis perdas amostrais e uma prevalência de uso de álcool em gestantes de serviços de atenção primária no Brasil de 6,1\% $\%^{26}$.

As gestantes foram recrutadas na unidade de assistência integral à saúde da mulher após anuência do diretor responsável. Inicialmente, todas as mulheres potencialmente elegíveis foram convidadas a participar do estudo antes das consultas pré-natais agendadas até composição da amostra necessária. A seguir, foram entrevistadas, face a face, em local privativo na instituição, por profissionais de saúde previamente treinados. Todas as participantes assinaram o Termo de Consentimento Livre Esclarecido, após elucidação dos objetivos, métodos, benefícios e possíveis riscos da pesquisa. Mulheres com idade inferior a 18 anos, além da obtenção do consentimento dos pais ou responsáveis, assinaram o Termo de Assentimento Livre e Esclarecido.

Utilizou-se um instrumento estruturado composto de características sociodemográficas e gestacionais, consumo de álcool durante a 
gestação atual e potenciais fatores associados ao evento de interesse. O questionário foi elaborado por especialistas em saúde mental e baseado em variáveis descritas e validadas em estudos semelhantes ${ }^{1,5,6,8,11,13,18,20,24,26}$.

A variável dependente analisada foi o autorrelato uso de álcool na gestação atual, categorizado em não ou sim. As variáveis independentes analisadas foram: idade (anos), escolaridade (anos), idade gestacional (semanas), estado civil (solteira versus [vs.] casada), antecedentes familiares de alcoolismo (não vs. sim), presença de hipertensão arterial sistêmica (HAS) e/ou diabetes pré-gestacional ou gestacional (não vs. sim), gravidez atual planejada (não vs. sim), antecedente pessoal de transtorno mental (não vs. sim), ideação suicida nos últimos 30 dias (não vs. sim), antecedente de aborto (não vs. sim), uso de tabaco nos últimos 30 dias (não vs. sim), antecedentes de IST no último ano (não vs. sim) e disfuncionalidade familiar (não vs. sim).

As variáveis: presença de IST na vida, aborto prévio e presença de doenças crônicas não transmissíveis (HAS e diabetes) foram verificadas no cartão da gestante ou em caso de ausência desses dados por meio do autorelato. A presença de disfuncionalidade familiar foi verificada por meio do instrumento APGAR de família, recomendado pela Organização Mundial de Saúde ${ }^{27}$ e Ministério da Saúde ${ }^{28}$, e validado previamente no Brasil ${ }^{29,30}$. Esse instrumento, desenvolvido por Smilkstein ${ }^{31}$ em 1978 permite um diagnóstico da disfuncionalidade familiar por meio de cinco perguntas relacionadas à satisfação do indivíduo sobre a família sobre os seguintes aspectos: adaptação, companheirismo, desenvolvimento e capacidade resolutiva. Para cada item, um escore que varia de zero a 10 pontos é atribuído escores que variam de zero a três pontos, gerando um escore total que varia de zero a 10 pontos. Pontuação inferior a sete sugere presença de disfuncionalidade familiar.

Os dados foram analisados no programa STATA, versão 12.0. Prevalência de consumo de álcool na gestação atual foi estimada com IC 95\%. Alfa de Crombach foi utilizado para avaliar a confiabilidade do instrumento APGAR de família. Análise de regressão de Poisson com variância robusta $^{32}$ foi utilizada para analisar os fatores associados ao desfecho investigado. Os testes quiquadrado $\left(\chi^{2}\right)$ e exato de Fisher foram utilizados para verificar as diferenças entre as proporções e o teste $\mathrm{t}$ de student para comparar médias na análise bivariada. A seguir, variáveis com $\mathrm{p}<0,10$ foram submetidas ao modelo de regressão múlti- pla. Valores de $\mathrm{p}<0,05$ foram considerados estatisticamente significantes.

Esse estudo foi aprovado pelo Comitê de Ética em Pesquisa da Universidade Federal de Goiás e respeitou os princípios éticos da Resolução 466/12 do Conselho Nacional de Saúde.

\section{Resultados}

Um total de 361 gestantes foi recrutado. Dessas, 27 recusaram a participar do estudo, resultando em uma amostra de 334 gestantes investigadas (taxa de resposta de 92,5\%). As médias de idade, escolaridade e idade gestacional foram de 24,4 anos (desvio padrão $[\mathrm{DP}] \pm 5,9$ ), 10,4 anos (DP $\pm 3,1$ ) e 21,7 semanas ( $\mathrm{DP} \pm 11,3$ ), respectivamente (Tabela 1).

Do total de participantes, 68,6\% (IC 95\%: $63,4-73,3 \%$ ) fizeram uso de álcool alguma vez na vida e 17,7\% (IC 95\%: 14,1-22,0\%) reportaram uso de álcool na gestação atual. As taxas de gestantes com antecedentes de HAS, diabetes, de IST e de aborto foram de 5,7\%, 3,3\%, 3,3\%, 11,0\%, respectivamente. Ideação suicida e uso de tabaco nos últimos 30 dias foi relatado por 9,3\% e 15,5\% das mulheres, respectivamente. Aproximadamente $19,9 \%$ das gestantes apresentaram escores compatíveis com a disfuncionalidade familiar no APGAR de família. Esse instrumento apresentou boa confiabilidade na amostra investigada (Alfa de Crombach: 0,851).

A Tabela 1 mostra a análise bivariada dos fatores associados ao consumo de álcool na gestação atual. Verificou-se maior proporção de uso de álcool em gestantes com autorrelato de diabetes gestacional ou pré-gestacional $(\mathrm{p}=0,01)$, com antecedente de IST ( $p=0,01)$, com disfuncionalidade familiar ( $p=0,03)$, com ideação suicida nos últimos 30 dias $(\mathrm{p}<0,001)$ e que faziam uso de tabaco $(p<0,001)$. Essas variáveis e antecedente de aborto $(p=0,09)$ foram incluídas no modelo de regressão múltipla.

Em análise multivariável, os fatores associados ao uso de álcool na gestação atual foram: idade (RP ajustada [RPaj]: 1,$06 ; p=0,03$; diabetes (RPaj: 1,24; $p=0,03$ ), ideação suicida (RPaj: 1,19; $p=0,03)$ e uso de tabaco (RPaj: 1,37 ; $<<0,001$ ) (Tabela 2).

\section{Discussão}

O presente estudo analisou a prevalência e fatores associados ao uso de álcool em uma amostra 
Tabela 1. Análise bivariada dos fatores associados ao consumo de álcool durante a gestação.

\begin{tabular}{|c|c|c|c|c|}
\hline \multirow{2}{*}{ Variáveis } & \multirow{2}{*}{ Total $^{\mathbf{a}}$} & \multicolumn{2}{|c|}{ Uso de álcool na gestação atual } & \multirow{2}{*}{$\mathbf{p}$} \\
\hline & & Positivo & $\%(\text { IC 95\%) })^{c}$ & \\
\hline Idade (anos) & $24,4(5,9)$ & & $6,7)$ & 0,91 \\
\hline Escolaridade (anos) & $10,4(3,1)$ & & $3,0)$ & 0,49 \\
\hline Idade gestacional (semanas) & $21,7(11,3)$ & & $1,8)$ & 0,61 \\
\hline \multicolumn{5}{|l|}{ Estado civil } \\
\hline Casada & 257 & 45 & $17,5(13,4-22,6)$ & 0,89 \\
\hline Solteira & 77 & 14 & $18,2(11,2-28,2)$ & \\
\hline \multicolumn{5}{|l|}{$\begin{array}{l}\text { Antecedente familiar de } \\
\text { alcoolismo }\end{array}$} \\
\hline Não & 184 & 27 & $14,7(10,3-20,5)$ & 0,11 \\
\hline Sim & 150 & 32 & $21,3(15,5-28,6)$ & \\
\hline \multicolumn{5}{|l|}{ Hipertensão Arterial Sistêmica } \\
\hline Não & 315 & 56 & $17,8(14,0-22,4)$ & 1,00 \\
\hline Sim & 19 & 3 & $15,8(5,5-37,6)$ & \\
\hline \multicolumn{5}{|l|}{ Diabetes } \\
\hline Não & 323 & 54 & $16,7(13,0-21,2)$ & 0,01 \\
\hline Sim & 11 & 5 & $45,5(21,3-72,0)$ & \\
\hline \multicolumn{5}{|l|}{ Gravidez atual planejada } \\
\hline Sim & 134 & 24 & $17,9(12,3-25,3)$ & 0,94 \\
\hline Não & 199 & 35 & $17,6(12,9-23,5)$ & \\
\hline \multicolumn{5}{|l|}{$\begin{array}{l}\text { Antecedente pessoal de } \\
\text { transtornos mentais }\end{array}$} \\
\hline Não & 293 & 51 & $17,4(13,5-22,2)$ & 0,74 \\
\hline Sim & 41 & 8 & $19,5(10,3-34,0)$ & \\
\hline \multicolumn{5}{|l|}{ Antecedente de IST ${ }^{\mathrm{b}}$} \\
\hline Não & 323 & 54 & $16,7(13,0-21,2)$ & 0,01 \\
\hline Sim & 11 & 5 & $45,5(21,3-72,0)$ & \\
\hline \multicolumn{5}{|l|}{ Antecedentes de aborto } \\
\hline Sim & 36 & 10 & $27,8(16,0-44,0)$ & 0,09 \\
\hline Não & 298 & 49 & $16,4(12,7-21,1)$ & \\
\hline \multicolumn{5}{|l|}{ Disfuncionalidade familiar } \\
\hline Não & 261 & 40 & $15,3(11,5-20,2)$ & 0,03 \\
\hline Sim & 72 & 19 & $26,4(17,6-37,6)$ & \\
\hline \multicolumn{5}{|l|}{ Ideação suicida } \\
\hline Não & 303 & 46 & $15,2(11,6-19,7)$ & $<0,001$ \\
\hline Sim & 31 & 13 & $41,9(26,4-59,2)$ & \\
\hline \multicolumn{5}{|l|}{ Uso de tabaco } \\
\hline Não & 282 & 29 & $10,3(7,3-14,4)$ & $<0,001$ \\
\hline Sim & 52 & 30 & $57,7(44,2-71,1)$ & \\
\hline
\end{tabular}

Nota: variáveis quantitativas são apresentadas como médias e desvio-padrão; a.Número de respostas válidas; b. Infecções Sexualmente Transmissíveis; c. Intervalo de confiança de 95\%.

de gestantes, contribuindo para o conhecimento dos determinantes do consumo dessa substância nessa população. Do nosso conhecimento, essa investigação apresenta os primeiros dados sobre a epidemiologia do álcool em gestantes de Goiás, Região Centro-Oeste do Brasil. Os resultados evidenciaram elevada prevalência de uso de álcool durante a gestação e sua associação com diabetes, ideação suicida e consumo de tabaco.
A prevalência do uso de álcool durante a gestação (17,7\%; IC 95\%: 14,1-22,0\%) foi superior à estimada em gestantes dos EUA $(10,2 \%)^{7}$ e Canadá $(10,8 \%)^{8}$. Além das diferenças metodológicas e amostragem entre os estudos, alguns fatores como características sociodemográficas, nível de desenvolvimento econômico, efetividade das políticas públicas de saúde e disparidades culturais podem contribuir para desigualdades na 
Tabela 2. Análise de regressão múltipla dos fatores associados ao consumo de álcool durante a gestação.

\begin{tabular}{lccc}
\hline \multicolumn{1}{c}{ Variáveis } & \multicolumn{2}{c}{ Razão de prevalência } & \multirow{2}{*}{$\mathbf{p}$} \\
\cline { 2 - 3 } & Não ajustada (IC 95\%) & Ajustada (IC95\%) & \\
\hline Idade (anos) & $1,06(0,99-1,13)$ & $1,06(1,00-1,13)$ & $\mathbf{0 , 0 3}$ \\
Diabetes & $1,24(1,01-1,53)$ & $1,24(1,01-1,51)$ & $\mathbf{0 , 0 3}$ \\
Antecedente de IST & $1,24(1,01-1,53)$ & $1,11(0,87-1,41)$ & 0,40 \\
Antecedente de aborto & $0,91(0,80-1,02)$ & $0,90(0,81-1,00)$ & 0,05 \\
Disfuncionalidade familiar & $1,09(1,00-1,19)$ & $1,00(0,92-1,09)$ & 0,89 \\
Ideação suicida & $1,23(1,08-1,39)$ & $1,19(1,04-1,35)$ & $\mathbf{0 , 0 3}$ \\
Uso de tabaco & $1,42(1,30-1,56)$ & $1,37(1,24-1,52)$ & $<\mathbf{0 , 0 0 1}$ \\
\hline
\end{tabular}

* Modelo ajustado por idade, antecedente familiar de alcoolismo, diabetes, antecedentes de aborto, APGAR de família, ideação suicida e uso de tabaco na gestação atual; a. Intervalo de confiança de $95 \%$

epidemiologia do uso dessa substância entre os países $^{33}$. Em geral, em países mais desenvolvidos como os EUA, o consumo de álcool é visto de forma mais negativa do que em culturas dos países da América Latina ${ }^{34}$, o que pode contribuir para menores índices de uso dessa substância.

No Brasil, a prevalência do uso de álcool na gestação varia conforme localização geográfica. Estudos evidenciam taxas de 6,1\% em Maringá $(\text { Paraná })^{26}, 22,3 \%$ em São Luís (Maranhão) ${ }^{35}$, 23,1\% em Uberlândia (Minas Gerais) ${ }^{24}, 7,4 \%$ a $40,6 \%$ no Rio de Janeiro ${ }^{36,37}$ e $23,0 \%$ a $33,3 \%$ em São Paulo ${ }^{23,25}$. Assim, a prevalência encontrada em Goiás encontra-se dentro da variação evidenciada em estudos conduzidos em gestantes de outras regiões do País.

Nessa investigação, o uso de tabaco foi associado ao consumo de álcool na gestação, assim como evidenciado na literatura nacional e internacional ${ }^{12,23,38}$. De fato, mulheres que fazem uso regular de tabaco na gravidez possuem maior vulnerabilidade ao consumo de álcool, o que potencializa o risco de desenvolvimento de eventos adversos gestacionais e efeitos negativos para o feto $^{1,38}$. O uso simultâneo de tabaco e álcool pode ser explicado pela legalidade e ampla disponibilidade dessas substâncias ${ }^{39}$, valores e significados culturais similares $^{7}$ e fatores de risco comuns ${ }^{12,38}$. Além disso, estudos têm identificado moléculas cerebrais, como neurotransmissores e receptores de nicotina, que fazem interação com ambas substâncias e medeiam o efeito e a sensibilidade dessas drogas no cérebro ${ }^{40}$.

Verificou-se associação entre ideação suicida nos últimos 30 dias e uso de álcool. Mulheres no período gravídico sofrem mudanças psicológicas, físicas e hormonais, tornado-as vulneráveis a agravos psiquiátricos ${ }^{41}$ e consumo de substâncias psicoativas, fatores de risco para ideação suicida.
O uso de álcool agrava traços na personalidade negativos (por ex.: aumento da impulsividade e agressividade), propicia eventos negativos na vida (por ex.: separação conjugal e isolamento social) e aumenta o risco de comorbidades psiquiátricas (por ex.: depressão), o que aumenta o risco de comportamentos suicidas ${ }^{42,43}$. Uma recente meta-análise mostrou que desordens relacionadas ao uso de álcool apresentam forte associação com ideação suicida (Odds ratio [OR]: 1,86), tentativas de suicídio (OR: 3,13 ) e suicídios (OR: 2,59) ${ }^{44}$. Nesse contexto, estratégias que visem à redução do uso de álcool na gestação podem ter impacto, além da diminuição de agravos materno-fetais associados, na redução de taxas de comportamentos suicidas nessa população.

No presente estudo, autorrelato de diabetes gestacional ou pré-gestacional foi associado ao uso de álcool na gestação atual. Aproximadamente $90 \%$ dos casos novos de diabetes tipo 2 podem ser atribuídos a fatores relacionados ao estilo de vida, incluindo o consumo de álcool ${ }^{45}$. Apesar da natureza transversal não permitir o estabelecimento da relação de causa e efeito, o uso e abuso de álcool representa um dos principais fatores de risco para diabetes mellitus, uma vez que tem efeito no pâncreas, interferindo no sistema metabólico e levando à resistência insulíni$\mathrm{ca}^{46}$. Nesse contexto, intervenções intensivas que visem promoção de estilo de vida saudável, incluindo diminuição do uso do álcool, pode retardar ou impedir a ocorrência de diabetes ${ }^{45}$, doença com graves conseqüências materno-fetais.

Nessa investigação, IST não permaneceu associado ao uso de álcool após ajuste por outras variáveis. Dados da literatura apontam uma forte associação entre consumo de álcool e IST ${ }^{21,47}$. Apesar da natureza transversal do estudo não permitir inferências causais, o uso dessa substân- 
cia potencializa o risco de aquisição dessas infecções, pois aumenta a chance de comportamentos de risco, como uso inconsistente do preservativo e diminuição da capacidade de negociação do uso do mesmo, além de contribuir para aumento da susceptibilidade biológica do organismo à IST $^{21}$. Por outro lado, a um diagnóstico de IST pode contribuir para aumento da taxa de uso de álcool durante a gestação, pois gera estresse relacionado à exposição da divulgação da doença, transmissão de IST ao feto e medo da morte ${ }^{48}$.

Essa investigação encontrou associação entre disfuncionalidade familiar e uso de álcool na gestação na análise bivariada, porém essa associação não se manteve após ajuste por outras variáveis. Há uma lacuna na literatura nacional e internacional sobre a relação entre a disfuncionalidade familiar e consumo de álcool em gestantes, utilizando instrumentos validados como o APGAR de família, sugerindo a necessidade de estudos futuros para verificar essa intersecção. No entanto, algumas evidências mostram que indivíduos pertencentes a famílias disfuncionais e com problemas familiares (por exemplo: conflitos parentais) apresentam maiores chances de envolvimento em comportamentos de risco para a saúde, incluindo o uso de substâncias ${ }^{49}$. Por exemplo, no México, um estudo conduzido em mulheres em idade reprodutiva encontrou uma associação entre uso de álcool e disfuncionalidade familiar no APGAR de família (OR: 2,98; $\mathrm{p}=0,001)^{50}$.

Esse estudo apresenta algumas limitações que devem ser levadas em consideração na interpretação dos seus resultados. A natureza transversal da investigação não permite estabelecimento de relações de causa e efeito entre o evento e variáveis investigadas. Desenhos longitudinais são mais indicados para verificar relação entre preditores e o consumo de álcool em gestantes, além de possibilitar o acompanhamento de tendências ao longo do tempo ${ }^{51}$. Os dados foram autorrelatados, passíveis de vieses de resposta e memória. A prevalência pode estar subestimada, uma vez que a medida do desfecho foi baseada no autorrelato $^{52}$ e o fato do uso de álcool ser socialmente menos aceito em mulheres. No entanto, a detecção dessa substância em exames laboratoriais de sangue e/ou urina é muito difícil e limitada, por causa da meia-vida curta do álcool, podendo, também, subestimar os resultados ${ }^{53}$. Os resultados não podem ser generalizados para gestantes de outras localidades geográficas, pois só considerou gestantes atendidas em um serviço de assistência ginecológica e pré-natal. Apesar das limitações, o estudo mostrou fatores com estreita relação ao uso de álcool, podendo contribuir para estratégias de redução e agravos relacionados ao uso dessa substância em gestantes.

Em conclusão, o estudo apontou elevada prevalência de uso de álcool na gestação atual. Além disso, verificou-se associação entre diabetes pré-gestacional ou gestacional, ideação suicida e uso de tabaco. A intersecção entre uso de álcool e variáveis como diabetes e ideação suicida em gestantes necessita de estudos mais robustos e devem ser alvos de investigações mais detalhadas, uma vez que existem poucos estudos sobre essa relação. Além disso, o instrumento APGAR de família deve fazer parte da avaliação em gestantes, pois a disfuncionalidade pode funcionar como mediador do uso de álcool nessa população.

Esse estudo apresenta importantes implicações na prevenção do uso de álcool em gestantes. Sabe-se que não há um nível seguro do uso de álcool em qualquer fase da gestação, devendo-se evitar qualquer tipo de álcool nesse período ${ }^{7}$. Assim, sugere-se o rastreio do padrão de consumo de álcool entre mulheres em idade fértil como rotina na área de saúde da mulher, planejamento familiar e pré-natal, com objetivo de verificar situação epidemiológica e traçar medidas de intervenção. Além disso, ações como aconselhamentos sobre os problemas associados ao uso dessa substância principalmente no pré-natal podem contribuir para redução efetiva ou anulação do uso de álcool em gestantes e agravos maternofetais relacionados. Em especial, considera-se que os serviços de atenção primária a saúde e de assistência pré-natal possuem papel impar nessas intervenções, incluindo triagem de consumo em todas as gestantes cadastradas, educação em saúde e encaminhamentos para serviços especializados. Por fim, determinantes associados ao consumo de álcool na gestação devem ser levados em consideração por profissionais de saúde na assistência integral às gestantes. 


\section{Referências}

1. Skagerstróm J, Chang G, Nilsen P. Predictors of drinking during pregnancy: a systematic review. $J$ Womens Health (Larchmt) 2011; 20(6):901-913.

2. Adusi-Poku Y, Bonney AA, Antwi GD. Where, when and whattype of alcohol do pregnant women drink? Ghana Med J 2013; 47(1):35-39.

3. Nolen-Hoeksema S. Gender differences in risk factors and consequences for alcohol use and problems. Clin Psychol Rev 2004; 24(8):981-1010.

4. O'Brien JW, Hill SY. Effects of prenatal alcohol and cigarette exposure on offspring substance use in multiplex, alcohol-dependent families. Alcohol Clin Exp Res 2014; 38(12):2952-2961.

5. Polańska K, Jurewicz J, Hanke W. Smoking and alcohol drinking during pregnancy as the risk factors for poor child neurodevelopment - A review of epidemiological studies. Int J Occup Med Environ Health 2015; 28(3):419-443.

6. Skagerström J, Alehagen S, Häggström-Nordin E, Arestedt K, Nilsen P. Prevalence of alcohol use before and during pregnancy and predictors of drinking during pregnancy: a cross sectional study in Sweden. BMC Public Health 2013; 13:780.

7. Tan CH, Denny CH, Cheal NE, Sniezek JE, Kanny D. Alcohol use and binge drinking among women of childbearing age - United States, 2011-2013. MMWR Morb Mortal Wkly Rep 2015; 64(37):1042-1046.

8. Walker MJ, Al-Sahab B, Islam F, Tamim H. The epidemiology of alcohol utilization during pregnancy: an analysis of the Canadian Maternity Experiences Survey (MES). BMC Pregnancy Childbirth 2011; 11:52.

9. O'Keeffe LM, Kearney PM, McCarthy FP, Khashan AS, Greene RA, North RA, Poston L, McCowan LM, Baker PN, Dekker GA, Walker JJ, Taylor R, Kenny LC. Prevalence and predictors of alcohol use during pregnancy: findings from international multicentre cohort studies. BMJ Open 2015; 5(7):e006323.

10. Petersen Williams P, Jordaan E, Mathews C, Lombard C, Parry CD. Alcohol and Other Drug Use during Pregnancy among Women Attending Midwife Obstetric Units in the Cape Metropole, South Africa. Adv Prev Med 2014; 2014:871427.

11. Míguez H, Magri R, Suárez M. Consumo de tabaco y bebidas alcohólicas durante el embarazo. Acta Psiquiatr Psicol Am Lat 2009; 55(2):76-83. param da elaboração do projeto, interpretação dos dados e escrita da versão inicial. RA Guimarães participou da análise estatística, interpretação dos dados, escrita da versão inicial e revisão da versão final. Todos os autores aprovaram a versão final a ser publicada.
12. López MB, Filippettii VA, Cremonteii M. Alcohol consumption before and during pregnancy in Argentina: prevalence and risk factors. Rev Panam Salud Publica 2015; 37(4-5):211-217.

13. Kroeff LR, Mengue SS, Schimidt MI, Duncan BB, Favaretto ALF. Fatores associados ao fumo em gestantes avaliadas em cidades brasileiras. Rev Saude Publica 2004; 38(2):261-267.

14. Isaksen AB, Østbye T, Mmbaga BT, Daltveit AK. Alcohol consumption among pregnant women in Northern Tanzania 2000-2010: a registry-based study. BMC Pregnancy Childbirth 2015; 15:205.

15. Lepper LE, Lluka A, Mayer A, Patel N, Salas J, Xaverius PK. Socioeconomic Status, Alcohol Use, and Pregnancy Intention in a National Sample of Women. Prev Sci 2016; 17(1):24-31.

16. Lanting CI, van Dommelen P, van der Pal-de Bruin KM, Bennebroek Gravenhorst J, van Wouwe JP. Prevalence and pattern of alcohol consumption during pregnancy in the Netherlands. BMC Public Health 2015; 15:723.

17. Van der Pol P, Liebregts N, de Graaf R, Korf DJ, van den Brink W, van Laar M. Facilitators and barriers in treatment seeking for cannabis dependence. Drug Alcohol Depend 2013; 133(2):776-780.

18. Zelner I, Matlow JN, Natekar A, Koren G. Synthesis of fatty acid ethyl esters in mammalian tissues after ethanol exposure: a systematic review of the literature. Drug Metab Rev 2013; 45(3):277-299.

19. Kwon M, Yang S, Park K, Kim DJ. Factors that affect substance users' suicidal behavior: a view from the Addiction Severity Index in Korea. Ann Gen Psychiatry 2013; 12(1):35.

20. Ethen MK, Ramadhani TA, Scheuerle AE, Canfield MA, Wyszynski DF, Druschel CM, Romitti PA; National Birth Defects Prevention Study. Alcohol consumption by women before and during pregnancy. Matern Child Health J 2009; 13(2):274-285.

21. Vagenas P, Lama JR, Ludford KT, Gonzales P, Sanchez J, Altice FL. A systematic review of alcohol use and sexual risk-taking in Latin America. Rev Panam Salud Publica 2013; 34(4):267-274.

22. Santos RR, Sundefeld MLMM, Garbin CAD, Moimaz SAD. Álcool, fumo e problemas periodontais em gestantes atendidas no sistema público de saúde. Revista Brasileira de Pesquisa em Saúde 2012; 14(2):38-43. 
23. Sbrana M, Grandi C, Brazan M, Junquera N, Nascimento MS, Barbieri MA, Bettiol H, Cardoso VC. Alcohol consumption during pregnancy and perinatal results: a cohort study. Sao Paulo Med J 2016; 134(2):146-152.

24. Souza LHRF, Santos MC, Oliveira LCM. Padrão do consumo de álcool em gestantes atendidas em um hospital público universitário e fatores de risco associados. Rev Bras Ginecol Obstet 2012; 34(7):296-303.

25. Mesquista MA, Segre CAP. Freqüência dos efeitos do álcool no feto e padrão de consumo de bebidas alcoólicas pelas gestantes de Maternidade pública da cidade de São Paulo. Rev Bras Crescimento Desenvolvimento Hum 2009; 19(1):63-77.

26. Kassada DS, Marcon SS, Pagliarini MA, Rossi RM. Prevalência do uso de drogas de abuso por gestantes. Acta Paul Enferm 2013; 26(5):467-471.

27. World Health Organization (WHO). Latino families and youth: a compendium of assessment tools. Geneva: WHO; 2012 [acesso 2016 fev 23]. Disponível em: http://www.rehueong.com.ar/sites/default/files/Binder -orpinas\%20meassurement.pdf

28. Brasil. Ministério da Saúde (MS). Envelhecimento e saúde da pessoa idosa. Brasília: MS; 2007. [acesso 2016 Fev 23]. Disponível em: http://bvsms.saude.gov.br/bvs/ publicacoes/abcad19.pdf

29. Duarte YAO. Família: rede de suporte ou fator estressor: a ótica de idosos e cuidadores familiares [tese]. São Paulo: Universidade de São Paulo; 2001.

30. Silva MD, Victor JF, Mota FRN, Soares ES, Leite BMB, Oliveira ET. Análise das propriedades psicométricas do APGAR de família com idosos do nordeste brasileiro. Esc Anna Nery 2014; 18(3):527-532.

31. Smilkstein G. The family APGAR a proposal for a family function test and its use by physicians. J Fam Pract 1978; 6(6):1231-1239.

32. Barros AJ, Hirakata VN. Alternatives for logistic regression in cross-sectional studies: an empirical comparison of models that directly estimate the prevalence ratio. BMC Med Res Methodol 2003; 3:21.

33. World Health Organization (WHO). Global status report on alcohol and health 2014. Geneva: WHO; 2014. [acesso 2016 Fev 23]. Disponível em: http://apps.who. int/iris/bitstream/10665/112736/1/9789240692763_ eng.pdf

34. Laranjeira R, Pinsky I, Sanches M, Zaleski M, Caetano R. Alcohol use patterns among Brazilian adults. Rev Bras Psiquiatr 2010; 32(3):231-241.

35. Rocha PC, Britto e Alves MT, Chagas DC, Silva AA, Batista RF, Silva RA. Prevalência e fatores associados ao uso de drogas ilícitas em gestantes da coorte BRISA. Cad Saude Publica 2016; 32(1):e00192714.

36. Freire K, Padilha PC, Saunders C. Fatores associados ao uso de álcool e cigarro na gestação. Rev Bras Ginecol Obstet 2009; 31(7):335-341.

37. Moraes CL, Reichenheim ME. Rastreamento de uso de álcool por gestantes de serviços públicos de saúde do Rio de Janeiro. Rev Saude Publica 2007; 41(5):695-703.

38. Lange S, Probst C, Quere M, Rehm J, Popova S. Alcohol use, smoking and their co-occurrence during pregnancy among Canadian women, 2003 to 2011/12. Addict Behav 2015; 50:102-109.

39. Funk D, Marinelli PW, Lê AD. Biological processes underlying co-use of alcohol and nicotine: neuronal mechanisms, cross-tolerance, and genetic factors. Alcohol Res Health 2006; 29(3):186-192.
40. Falk DE, Yi HY, Hiller-Sturmhöfel S. An epidemiologic analysis of co-occurring alcohol and tobacco use and disorders: findings from the National Epidemiologic Survey on Alcohol and Related Conditions. Alcohol Res Health 2006; 29(3):162-171.

41. Faisal-Cury A, Menezes P, Araya R, Zugaib M. Common mental disorders during pregnancy: prevalence and associated factors among low-income women in São Paulo, Brazil: depression and anxiety during pregnancy. Womens Ment Health 2009; 12(5):335-343.

42. Sher L. Alcohol consumption and suicide. QJM 2006; 99(1):57-61.

43. Brady J. The association between alcohol misuse and suicidal behaviour. Alcohol Alcohol 2006; 41(5):473478.

44. Darvishi N, Farhadi M, Haghtalab T, Poorolajal J. Alcohol-related risk of suicidal ideation, suicide attempt, and completed suicide: a meta-analysis. PLoS One 2015; 10(5):e0126870.

45. Chen L, Magliano DJ, Zimmet PZ. The worldwide epidemiology of type 2 diabetes mellitus-present and future perspectives. Nat Rev Endocrinol 2011; 8(4):228236.

46. Kim SJ, Kim DJ. Alcoholism and Diabetes Mellitus. Diabetes Metab J 2012; 36(2):108-115.

47. Cook RL, Clark DB. Is There an Association Between Alcohol Consumption and Sexually Transmitted Diseases? A Systematic Review. Sex Transm Dis 2005; 32(3):156-164.

48. Watt MH, Eaton LA, Choi KW, Velloza J, Kalichman SC, Skinner D, Sikkema KJ. "It's better for me to drink, at least the stress is going away": perspectives on alcohol use during pregnancy among South African women attending drinking establishments. Soc Sci Med 2014; 116:119-125.

49. Velleman RD, Templeton LJ, Copello AG. The role of the family in preventing and intervening with substance use and misuse: a comprehensive review of family interventions, with a focus on young people. Drug Alcohol Rev 2005; 24(2):93-109.

50. Boo-Vera D, Martínez-Torres J, Montesinos-Balboa JE. Influencia del consumo de alcohol en mujeres en la funcionalidad conyugal y familiar. Revista Salud Pública y Nutrición 2010; 11:2.

51. Hosseinpoor AR, Bergen N, Kunst A, Harper S, Guthold R, Rekve D, d'Espaignet ET, Naidoo N, Chatterji S. Socioeconomic inequalities in risk factors for non communicable diseases in low-income and middle-income countries: results from the World Health Survey. BMC Public Health 2012; 12:912.

52. Nelson DE, Naimi TS, Brewer RD, Roeber J. US state alcohol sales compared to survey data, 1993-2006. Addiction 2010; 105(9):1589-1596.

53. Wong S, Ordean A, Kahan M; Maternal Fetal Medicine Committee; Family Physicians Advisory Committee; Medico-Legal Committee; Society of Obstetricians and Gynaecologists of Canada. Substance use in pregnancy. J Obstet Gynaecol Can 2011; 33(4):367-384.

Artigo apresentado em 04/05/2016

Aprovado em 03/10/2016

Versão final apresentada em 05/10/2016 